\title{
Experimental Methods for Characterizing Dynamic Response of Soft Materials
}

\author{
Weinong Wayne Chen ${ }^{1}$
}

Received: 10 November 2015/ Accepted: 16 January 2016/Published online: 27 January 2016

(C) Society for Experimental Mechanics, Inc 2016

\begin{abstract}
Soft materials are widely used in applications subjected to impact loading but are less well characterized in terms of their constitutive behavior under high-rate loading conditions. A variety of modified Kolsky bar methods have been developed recently to obtain high-fidelity dynamic mechanical responses of soft materials. This article briefly summarizes major issues associated with the application of Kolsky bars in soft-material characterization, including weak transmitted signals, uniform loading on the specimen, constant strain rate deformation, intermediate strain rate experiments, specimen gripping methods, and inertia effects. Experimental remedies and remaining challenges are presented to address each of the issues encountered. Recent advances in real-time visualization of dynamic deformation and damage processes are also discussed.
\end{abstract}

Keywords Kolsky bar (split Hopkinson pressure bar or SHPB) - Soft materials - Dynamic constitutive behavior . Damage visualization

\section{Introduction}

Soft materials have low mechanical stiffness and strength. Polymers, polymeric and metallic foams, and biological tissues are typical examples of soft materials. Engineered soft materials, such as rubbers, are widely used for shock mitigation and vibration isolation. However, the dynamic

\section{Weinong Wayne Chen}

wchen@purdue.edu

1 Schools of Aero/Astro and Materials Engineering, Purdue University, West Lafayette, IN 47907, USA responses of this class of materials are interestingly less understood and modeled, compared to solid metallic and ceramic materials. One reason for this lack of understanding is the limitation in dynamic experimental methods to obtain reliable material behavior at high strain rates such that physical insights into the dynamic deformation and failure processes can be developed for physics-based modeling. To cross this research gap, development of highrate experimental methods have been a focus recently. Most of these techniques are based on Kolsky bars [1-5], also called split Hopkinson pressure bars (SHPB) [6, 7], which will be the focus of the discussions in this article.

Soft material is not a very precisely defined concept. From the Kolsky-bar-experiment point of view, when the strength of the specimen material is below about $10 \mathrm{MPa}$, issues associated with soft material characterization start to appear. Therefore, in Kolsky bar experiments, $10 \mathrm{MPa}$ strength may serve as a dividing line between soft and regular materials. Another possible definition is the ratio of bulk modulus over shear modulus. As will be discussed, when the bulk modulus is $4-5$ orders of magnitude higher than the shear modulus of the specimen material, it becomes very challenging to obtain dynamic material behavior by uniaxial compression or tension experiments [8].

Kolsky bars in various versions have been widely used to obtain families of stress-strain curves for engineering materials at high strain rates between $10^{2}$ and $10^{4} \mathrm{~s}^{-1}$ [7], and recently up to $10^{5} \mathrm{~s}^{-1}$ [9], since it was developed by Kolsky [6], including many soft materials [10-15]. However, when the strength of the soft material is below $10 \mathrm{MPa}$, the validity and accuracy of experimental results may have to be evaluated since the testing conditions in the soft specimens may differ significantly from those assumed or desired $[4,5,7]$. Modifications are necessary to the 
Kolsky bars to ensure the achievement of desired testing conditions on the specimens for accurate results. Also, recent integration of high-speed X-ray visualization techniques with the classical Kolsky bar dynamic loading methods opens up new potentials for better understanding of dynamic behavior of materials [16-21]. Besides the X-ray "see-through" imaging methods, high-speed fullfield surface measurements such as virtual field methods [22, 23] and digital image correlation [24, 25] provide valuable additional measurements on top of the traditional stress-strain curves. In this paper, we summarize these recent developments in Kolsky bar techniques for dynamic characterization of soft materials, with the emphasis on the developments over the last decade. The review is focused more on the research from Purdue University. We first briefly review the Kolsky bar, then illustrate the challenges faced with a soft specimen in the test section, summarize experimental solutions to address these challenges, point out the remaining issues, and finally introducing X-ray visualization methods.

\section{Kolsky Bar}

A Kolsky bar, or SHPB, schematically shown in Fig. 1, consists of a striker, an incident bar, and a transmission bar. The specimen is sandwiched between the incident and transmission bars. The impact of the striker onto the incident bar generates the stress wave needed to load the specimen dynamically. Strain gages on the incident and transmission bars quantify the stress waves propagating in these bars. One-dimensional elastic wave mechanics relates the waves to the dynamic load and deformation histories in the specimen $[6,7]$.

During a SHPB experiment, the incident and transmission bars serve as sensors of the experiment and thus need to meet some strict requirements for data acquisition. The bar material needs to be well understood for data interpretation. These bars must remain elastic since elastic wave theories are used to relate the bar surface measurements to the stress waves inside. If feasible, the bar lengths should be sufficiently long to avoid wave overlapping. Otherwise, wave decoupling techniques are needed [26, 27]. Slender bars help to minimize the effects of two-dimensional stress wave propagation in the bars. The ends of the bars in contact with the specimen must remain flat and parallel to ensure boundary conditions on the specimen, which is typically less an issue with soft specimens unless the bars are soft too.

One-dimensional elastic stress-wave analysis on the bars yields the engineering strain rate, strain, and stress histories in the specimen $[1,7]$,

$\dot{\varepsilon}=\frac{C_{0}}{L_{s}}\left[\varepsilon_{i}(t)-\varepsilon_{r}(t)-\varepsilon_{t}(t)\right]$

$\varepsilon=\frac{C_{0}}{L_{s}} \int_{0}^{t}\left[\varepsilon_{i}(t)-\varepsilon_{r}(t)-\varepsilon_{t}(t)\right] d t$

$\sigma=\frac{A_{0}}{2 A_{s}} E_{0}\left[\varepsilon_{i}(t)+\varepsilon_{r}(t)+\varepsilon_{t}(t)\right]$

where $\varepsilon_{i}(t), \varepsilon_{r}(t)$, and $\varepsilon_{t}(t)$ are the three measured quantities in a Kolsky-bar experiment: incident, reflected, and transmitted strain histories; $A_{0}$ is the cross-sectional area of the bars; $E_{0}$ and $C_{0}$ are Young's modulus and elastic bar wave speed in the bar material, respectively; $A_{s}$ and $L_{s}$ are initial cross-sectional area and length of the specimen, respectively. When the specimen is loaded uniformly, the axial stress on the two end faces are the same, which can be expressed in terms of the measured quantities as

$\varepsilon_{i}(t)+\varepsilon_{r}(t)=\varepsilon_{t}(t)$

Under the uniform loading assumption, Eqs. (1)-(3) are simplified as

$\dot{\varepsilon}=-2 \frac{C_{0}}{L_{s}} \varepsilon_{r}(t)$

$\varepsilon=-2 \frac{C_{0}}{L_{s}} \int_{0}^{t} \varepsilon_{r}(t) d t$

$$
\sigma=\frac{A_{0}}{A_{s}} E_{0} \varepsilon_{t}(t)
$$

Fig. 1 A schematic of conventional Kolsky bar setup

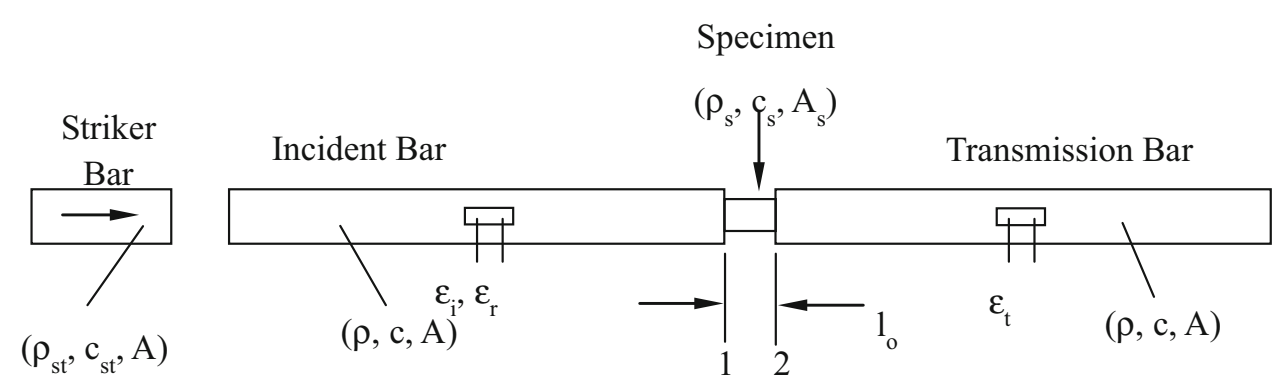


Elimination of time in (6) and (7) results in a series of dynamic stress-strain relations obtained at the strain rates calculated by (5). In addition to the widely-used compression bars, there are also tension and torsion Kolsky bars. The wave propagation analysis and the resultant data reduction methods are the same, just replacing compressive stress and strain with tension or torsion stresses and strains.

\section{Challenges and Remedies in Soft Material Characterization}

\section{Challenge: Weak Transmitted Signals}

Equation (7) indicates that the stress in the specimen is directly related to the transmitted signal $\varepsilon_{t}$ measured on the transmission bar. With a soft specimen in the test section of a Kolsky bar, this transmitted signal may be too weak to be accurately sensed by the regular resistor strain gages on a metallic transmission bar. The noise level may be too high such that the actual transmitted signal is embedded in the noise [28, 29]. For example, When a Plexiglas specimen is in the test section, its dynamic compressive strength is in the order of $100 \mathrm{MPa}$ and a background noise equivalent to $2 \%$ of the peak signal ( $2 \mathrm{MPa}$ ) is acceptable. However, when the peak stress of a specimen is in the order of a few $\mathrm{MPa}$, the noise is in the same order of magnitude starts to seriously affect the results. Furthermore, the peak stress of some soft tissues, such as brain and lung tissues, is in the order of $\mathrm{kPa}$, as will be discussed later. Conventional highrate uniaxial-stress experiments become infeasible. To accurately determine the weak signal transmitted from a soft specimen, the method of obtaining the transmitted signal in a Kolsky bar has to be modified to increase the sensitivity.

\section{Remedy: Weak Bar Signal Sensing Methods}

To measure the weak transmitted pulse with an acceptable signal-to-noise ratio, a number of methods have been utilized. These methods can be categorized into two types: enhancing bar surface strain amplitude and direct force measurements. The surface strain enhancement methods include reducing the transmission bar cross-sectional area, using a bar material with a lower Young's modulus, employing high-sensitivity strain gages, and combinations of these techniques. Direct force measurement methods include embedded piezoelectric sensors and force transducers.

An examination of Eq. (7) indicates that, under the condition of a fixed stress level and specimen cross-sectional area, the bar cross-sectional area and/or the bar
Young's modulus must be reduced to increase the amplitude of the transmitted strains. To reduce the bar crosssectional area, hollow tubes have been used as transmission bars [28]. In general, any method to reduce the cross-sectional area is acceptable. The incident and transmission bars in a Kolsky-bar setup do not need to be identical in cross-sectional area or material. However, when nonidentical bars are used, the data-reduction equations must be adjusted accordingly. If the bar material is the same, the axial engineering strain of the specimen is [28]

$$
\begin{aligned}
\varepsilon(t) & =\frac{c_{0}}{L} \int_{0}^{t}\left[\varepsilon_{i}(\tau)-\varepsilon_{r}(\tau)-\varepsilon_{t}(\tau)\right] \\
& =\frac{c_{0}}{L}\left(1-\frac{A_{i}}{A_{t}}\right) \int_{0}^{t} \varepsilon_{i}(\tau) d \tau-\frac{c_{0}}{L}\left(1+\frac{A_{i}}{A_{t}}\right) \int_{0}^{t} \varepsilon_{r}(\tau) d \tau
\end{aligned}
$$

where $A_{i}$ and $A_{t}$ are the cross-sectional areas of the solid incident and the hollow transmission bars, respectively. The stress in the specimen can still be calculated using Eq. (7). Equation (8) can be further expanded to account for different bar materials.

Using transmission bars made of materials with lower Young's moduli, such as aluminum, beryllium, titanium, and magnesium, can also increase the transmitted strain signal [1, 28-32]. For example, aluminum alloys have a Young's modulus about $1 / 3$ of that of a steel, under the same axial stress, the strain on the aluminum transmission bar surface is three times higher. If an aluminum tube is used, the surface strain is an order of magnitude higher than on a steel bar surface [7]. If a polymer transmission bar is used [33-40], the Young's modulus is an order of magnitude lower than most metallic bars, although viscoelastic analysis is needed to relate the surface strain to axial stress. However, the current strain measurement methods using strain gages limits the full utilization of this potential. Polymer bars are typically poor in heat conduction. This limits the exciting voltage of the Wheatstone bridges connecting the surface strain gages to an order of magnitude lower than that for gages on metal bar surfaces. However, recent application of interferometry techniques onto Kolsky bars [9] leads to the possibility of detecting the bar surface strain without relying on a strain gage. The advantage of the low elastic moduli of polymer bars may be fully utilized by using optical methods to measure bar strains, although the data interpretation is more elaborative due to the viscoelastic nature of the bars that relates the axial stress to the surface strain [39].

Using a more sensitive strain gage can also detect lowamplitude strain signals with a decent signal-to-noise ratio $[7,41]$. The gage factor of a resistor strain gage is only about 2, whereas the factor for a semi-conductor strain gage is 70 times higher. Thus, an aluminum tube with 
semiconductor strain gages on its surface is 700 times more sensitive than a steel bar, which is sufficient for sensing the weak signals transmitted from most of the soft specimens.

An alternate approach for sensing the weak transmitted signal is to measure the axial force directly with a force transducer. One method is to embed an X-cut quartz crystal disk in the middle of an aluminum transmission bar [42]. The mechanical impedance of the quartz crystal is within about $3 \%$ of that of aluminum. The quartz crystal is also a highly sensitive piezoelectric pressure transducer. The embedment of a quartz crystal allows the measurement of the transmitted force history without disturbing the onedimensional force transmission in the bar.

In some experiments with very weak specimens, e.g., a spider silk [43], the transmission bar hardly moves due to the small amplitude of the transmitted pulse. In this case, the transmission bar may be replaced by a load cell that measures the small force across the specimen directly [44]. This method has been recently extended to Kolsky bar experiments performed in laboratories with limited physical space [16].

\section{Challenge: Dynamic Stress Equilibrium}

In a Kolsky bar experiment, we obtain dynamic stressstrain response of the specimen material, which is assumed to be point-wise valid in the subsequent modeling efforts. However, the material behavior is obtained from a specimen of finite sizes, not a point. Therefore, the properties are the volume-averaged responses of the specimen. To ensure that the properties are true representatives of material responses, the specimen needs to be loaded uniformly such that the assumption expressed by Eq. (4) is valid. The load build-up in the specimen is through the multiple reflections of stress waves along the axial direction of the specimen. Uniform loading is achieved quickly in specimens with high stress-wave speeds such as most ceramics and metals. However, the stress state in a soft specimen may not be uniform over the entire loading duration in a Kolsky-bar experiment. For example, the stress-wave speed in a lung tissue is characterized by the elasticity of air bubbles and the density of water (blood). The wave speed is on the order of dozens of meters per second $[45,46]$. As will be described later, experimental challenges still exist for characterizing such extreme soft materials. Even in a less soft specimen, the stress-wave speed is still much less than those in ceramics or metals. The characteristic low wave speeds in soft specimens result in non-equilibrated stresses in the specimen causing nonuniform deformation, which invalidates the experimental results for material property characterization [29]. This calls for modifications to Kolsky bar experiments to achieve uniform loading.

\section{Remedy: Loading the Specimen Uniformly}

When the purpose of a Kolsky bar experiment is to obtain the dynamic properties of a material, the specimen must deform nearly uniformly such that the specimen response averaged over the specimen volume is a good representation of the pointwise-valid material property. In the Kolsky bar experiments, we do not have the means to ensure the specimen to deform homogeneously. However, we can load the specimen nearly uniformly, which may be checked by comparing the forces (or stresses) at both ends of specimen during an experiment. When the measurements at the two ends show nearly identical force histories, we call the specimen to be in dynamic stress equilibrium.

The stresses at the two ends of specimen may be calculated using a 2-wave and 1-wave method [47],

$\sigma_{1}=E_{0}\left(\varepsilon_{i}+\varepsilon_{r}\right)$

$\sigma_{2}=E_{0} \varepsilon_{t}$

where $\sigma_{1}$ and $\sigma_{2}$ are the stresses in the bars at the front and back ends of specimen, respectively. As long as the incident $\left(\varepsilon_{i}\right)$, reflected $\left(\varepsilon_{r}\right)$, and transmitted $\left(\varepsilon_{t}\right)$ signals are recorded, the stress equilibrium process can be monitored. However, when the specimen is a soft material, the low transmitted pulse results in nearly the identical reflected pulse $[29,33]$. This leads to high noise level in the calculation of stress at the front end of specimen using the difference between the incident and reflected pulses [Eq. (9)]. An alternate method is needed to evaluate stress equilibrium process in a soft material specimen.

One method to obtain the force (or stress) at the front end of specimen is direct measurement with a highly sensitive force transducer. Chen et al. [42] attached a pair of quartz crystal force transducers to the specimen ends of incident and transmission bars (Fig. 2). The quartz-crystal transducer itself may bring additional inertia stress into the recorded signal. Casem et al. [48] developed an inertiacompensation method to solve this problem through using three quartz-crystal transducers together. A comparison of the signals from the quartz crystal force transducers embedded at the bar ends provides a direct monitor of dynamic stress equilibrium process in specimen. Now the dynamic stress equilibrium in the specimen can be monitored. However, how to facilitate an equilibrium in the specimen is still a challenge in the design of Kolsky bar experiments on soft materials.

Dynamic stress equilibrium is achieved through stress wave reflections and transmissions in the specimen at the specimen/bar interfaces [49]. The wave speed of a material is thus an important parameter that determines the time to reach dynamic stress equilibrium. For a soft material, the 
Fig. 2 A schematic of the Kolsky bar modified for softmaterial characterization

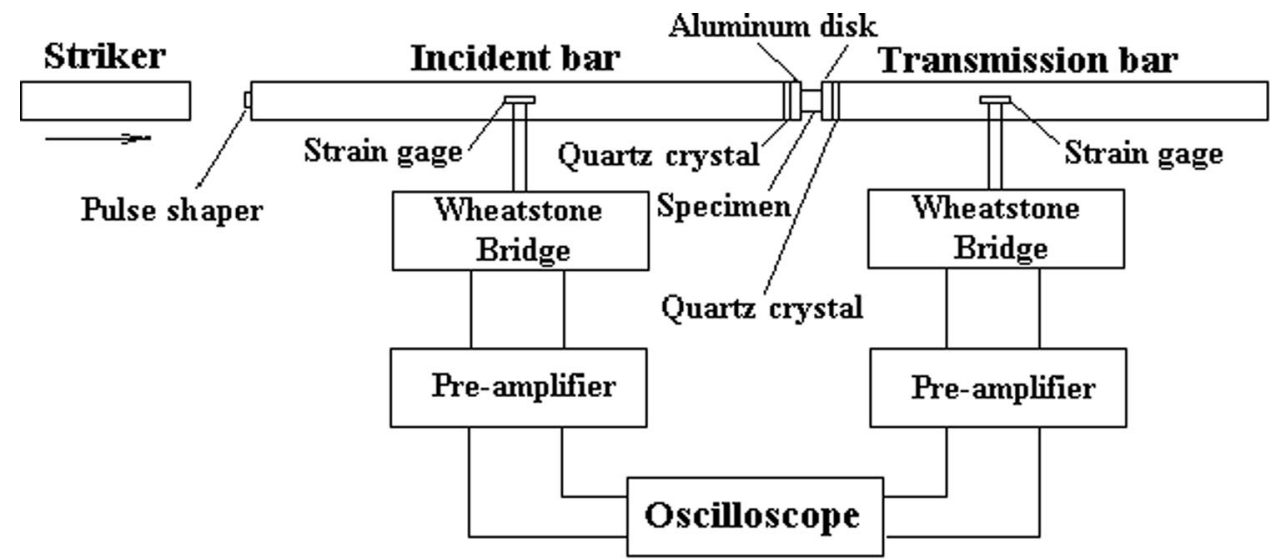

wave speed is typically very slow, which poses a challenge to achieve dynamic stress equilibrium in a Kolsky bar experiment. Another parameter that determines stress equilibrium is specimen thickness. A thin specimen is often used to shorten the time to dynamic stress equilibrium. A side benefit of using a thin specimen is the minimization of stress-wave attenuation through the specimen $[6,50,51]$. However, a thin specimen also brings in side effects of more significant end friction effects.

When the loading rate is very high, it has been demonstrated that, even though a thin specimen is used, the dynamic stress equilibrium may not be achieved [29]. Under such loading conditions, the soft specimen is an effective shock absorber, rather than a specimen to obtain material properties. Thus, the loading rate on the specimen must also be controlled for dynamic stress equilibrium [49]. The loading rate history in a specimen is controlled by pulse-shaping techniques, which have been extensively developed and documented [7, 52-54].

Unlike quasi-static testing machines, Kolsky bars do not have closed-loop control systems to ensure the specimen under desired testing conditions. Pulse shaping is about the only active control over the testing conditions on the specimen. The control over the incident pulse shapes may be achieved using explosive or electro-magnetic methods. However, the most commonly used method is mechanical [54]. A piece or a combination of plastically deformable materials, called a pulse shaper, is placed at the impact end of the incident bar, as shown in Fig. 3. When the striker impacts the incident bar through the pulse shaper, the plastic deformation on the pulse shaper gradually opens up the momentum passage from striker to the incident bar, thereby controlling the amplitude of the incident pulse over time. The desired pulse shaper is such that it can facilitate constant strain-rate deformation in the specimen under dynamic stress equilibrium over most of the duration of the experiment. A side gain of using a pulse shaper is the filtering of highfrequency components in the incident pulse, generating a nearly non-dispersive incident pulse that loads the specimen without severe oscillations in the loading history. The necessary pulse shaper geometry may be computed using published models [7, 54]. Examples of pulse shaper design are available [5]. In addition, a pulse shaper also minimizes initial inertia effects in the specimen, which is discussed later in the inertia effects section.

\section{Challenge: Constant Strain Rate}

The mechanical behavior of most soft materials, such as polymers and biological tissues are known to be strainrate sensitive [55]. To examine the strain-rate effects and to report the stress-strain response as a function of strain rates, the strain rate in a specific experiment should be close to a constant. In a typical Kolsky bar experiment, the time history of an incident pulse is a trapezoidal shape with a flat plateau over most of the duration. If the specimen is very soft such that its impedance is nearly negligible compared to that of the incident bar, the end of the incident bar moves at a nearly constant velocity under the loading of the stress-wave plateau, whereas the transmission bar hardly moves. This generates a constant engineering strain rate in the specimen. If the specimen is not that soft, a considerable fraction of the incident pulse will be transmitted through the specimen, leaving the reflected pulse with a decreasing amplitude as the specimen deforms. This leads to a non-constant engineering strain rate [Eq. (5)]. The incident pulse shape will have to be modified if a constant engineering strain rate is desired. On the other hand, if a constant true strain rate is intended, the incident pulse will also need to be adjusted such that a constant true strain rate in the specimen is achieved after the initial strain-rate rise from zero to the desired constant value. 
Fig. 3 Illustration of a pulse shaper at the impact end of the incident bar

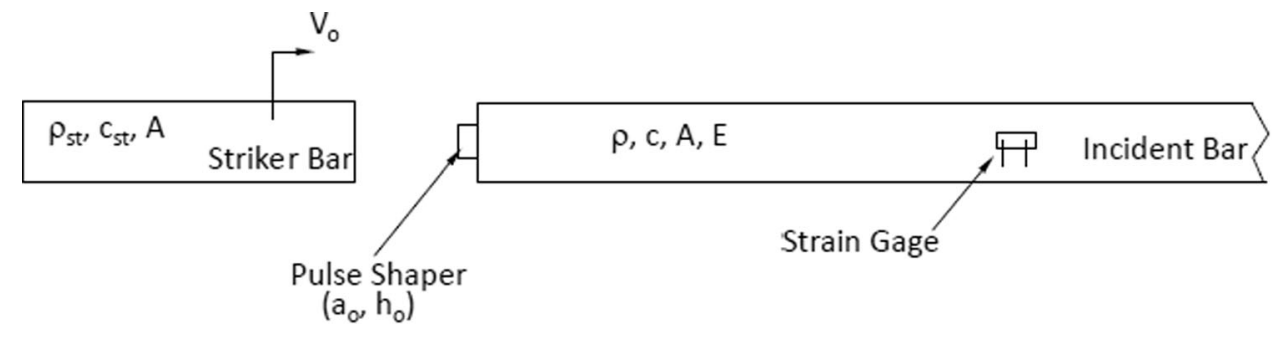

\section{Remedy: Pulse Shaping}

As just been discussed, pulse shaping is the method to control the time history of the loading pulse [52-54]. To conduct Kolsky bar experiments, either in compression or tension, on very soft materials for constant engineering strain rates, only a small piece of pulse shaper (e.g., a thin and small diameter annealed copper disk) is needed to generate a trapezoidal incident pulse without associated high-frequency oscillations. Such an incident pulse is nearly non-dispersive and loads the specimen in a welldefined manner [7]. In addition to the constant engineering strain rate in the specimen, the pulse shaping method also creates an incident pulse with an extended rise time to allow the specimen get into constant-rate deformation before being damaged. When a true constant strain rate is desired, which requires a decreasing-amplitude incident pulse over time, a tapered striker plus a small pulse shaper will be required to generate such incident pulses [56]. If the specimen is not very soft, which causes the engineering strain rate to decrease over time, and a constant engineering true strain rate is required, an increasing-amplitude incident pulse is needed. The pulse-shaping method to generate such an incident pulse is more conventional [5254].

\section{Challenge: Intermediate Strain Rates}

A challenge related to the constant strain-rate deformation is the achievement of strain rates lower than the typical Kolsky bar rate which starts from about $500 \mathrm{~s}^{-1}$. Many applications, such as vehicle crash or sports collision, encounter strain rates between a few dozen to a few hundred per second. However, when the strain rates in the experiments on soft materials are at such low levels (referred as intermediate strain-rate range), the overall strain in the specimen can hardly reach sufficiently high levels where yield or damage starts to occur in the soft materials, many of which are capable of large deformations [26], due to the limited load duration in typical Kolsky-bar experiments. This leaves scarcity of data in the intermediate strain-rate range of $10^{0}-10^{2}[26,55,57-60]$. Most conventional hydraulically-driven materials testing machines are designed to conduct quasi-static experiments at strain rates up to $10^{-1} \mathrm{~s}^{-1}$. On the other hand, typical Kolsky bars load specimens at strain rates above $10^{2} \mathrm{~s}^{-1}$. The mechanical response in the intermediate strain rate range between 1 and $5 \times 10^{2} \mathrm{~s}^{-1}$ is a much less explored range for any material, including soft materials. Drop-weight towers and Charpy tests subject the specimens to intermediate strain rates, but do not directly provide complete stress-strain curves as a function of strain rate. Therefore, for the needs in practical applications, it is necessary to develop reliable experimental methods to characterize mechanical responses of materials at intermediate strain rates. From the experimental technique point of view, it is also necessary to check if there are any system errors between quasi-static and Kolsky bar experimental techniques since the results from the two groups of experimental methods are presented on the same graph for extracting rate effects. Therefore, it is desired to overlap the strain rate ranges of quasi-static load frames and Kolsky bars.

\section{Remedy: Fast Hydraulic Loading Frames and Long Kolsky Bars}

To increase the loading rates in quasi-static load frames, high-rate commercial machines have been developed although the operations in the intermediate rate range are typically open-loop [61, 62]. Strain-gage types of load cells do not have sufficiently high frequency responses anymore at this rate range. Higher frequency response load cells are necessary $[60,63]$. When the specimen is soft, such a machine may run experiments at strain rates as high as $200 \mathrm{~s}^{-1}$ [57]. To conduct experiments at intermediate strain rates on soft materials with Kolsky bars, the necessary loading duration becomes a challenge. When the strain rate is constant, the overall strain in the specimen is approximately the strain rate multiplied by the loading duration. Since most soft specimens can deform to large strains, with the limit in the strain rates, the loading duration must be sufficiently long to deform the specimens to large strains. This long loading duration requires very long bars or bars with low wave speeds. Limited by physical lab size, Zhao and Gary [26, 27] utilized periodic round trips of 
a loading pulse in the incident bar to load the specimen progressively to large strains. They also developed a device composed of a hydraulic oil jack and a reservoir of compressed air to maintain the pressure to push the striker [26, 55]. When a limited length of Kolsky bar is used, the overlapped stress waves measured from bar surfaces should be properly separated $[26,35,55,64]$.

When lab space permits, a long Kolsky bar may be developed, as shown in Fig. 4, to conduct the experiments the same way as in regular Kolsky bars [57]. The data acquisition and reduction also does not involve added uncertainties from wave separation operations, which improves the accuracy of the experimental results. It has been demonstrated that the strain rate from a long bar can be as low as $40 \mathrm{~s}^{-1}$ [57].

\section{Challenge: Tensile Specimen Gripping}

As mentioned earlier, Kolsky bars can also be operated in tension mode. In experiments on solid materials, specimen gripping methods in the tensile testing section are well developed [65]. When the specimen is a soft material, such as a soft-tissue specimen under tension, gripping becomes an experimental challenge to avoid stress concentrations between the gripping area and the gage section [66, 67]. Even in a Kolsky bar compression experiment, the specimen initial length may be a challenge to be accurately determined $[68,69]$. In compression experiments on most engineered materials, the specimen is sandwiched between the incident and transmission bars. In the sample installation process, a slight axial force is applied such that the end faces of the specimen are in good contact with the bar end faces through lubricants. The adhesion of the lubricant layer, or friction when a lubricant is not used, on the

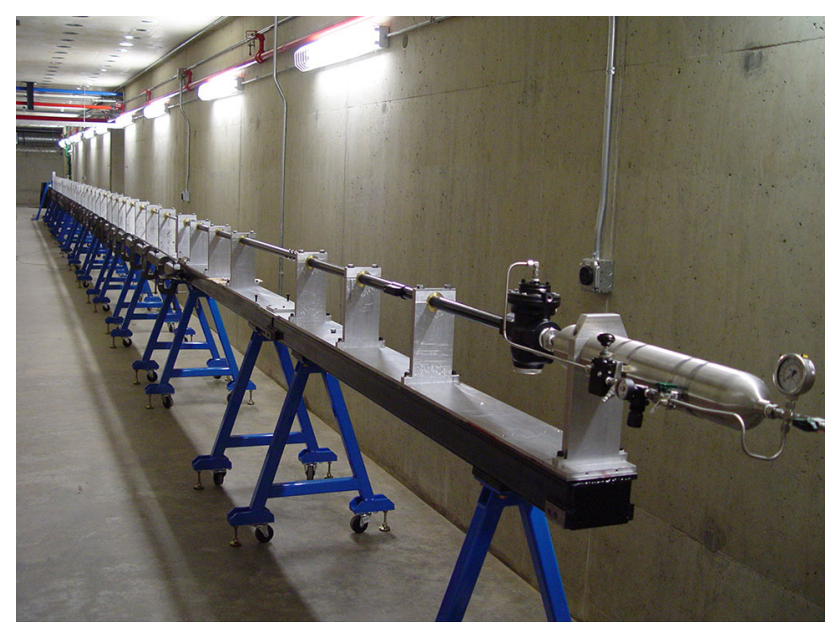

Fig. 4 Photograph of a long Kolsky bar designed to deform specimens to large strains at intermediate strain rates $\left(40-200 \mathrm{~s}^{-1}\right)$ interfaces keeps the specimen in place. If the specimen is a soft tissue, the initial axial pressure cannot be applied due to the very low stiffness of the tissue material. To obtain reliable experimental results, proper gripping methods must be designed to minimize non-uniform deformation, slippage and localized loading.

\section{Remedy: New Gripping Methods}

In compression experiments on soft tissues, the specimen geometry is typically far from as regular as engineered materials. In such circumstances, the thickness of the specimen needs to be measured using optical methods. A thickness gage adjusted to the individual specimen thickness is used to keep the incident and transmission bars apart at the correct distance such that there is no initial strain in the specimen. Without the initial axial pressure, the specimen will slip off the test section. A tiny rough spot can be introduced to one of the bar end surfaces to keep the specimen in position. Vegetable oil is often used as lubricant [68-71].

The specimen gripping issue becomes even more challenging when the experiments on soft materials are conducted in tension. Tensile specimen mounting is typically through gripping the surface at the ends of a specimen using a mechanical thread or chemical adhesion. A soft specimen has low shear stiffness. The fixed end surfaces cannot ensure a flat cross-section of a specimen to remain flat during tensile deformation. For a specimen with a certain thickness, gripping can only occur on the surfaces. The center portion of the cross-section will be pulled away along the tensile loading direction, leading to non-uniform deformation. When a soft biological tissue is in the tensile test section, no mechanical gripping can be used since any gripping pressure will squeeze the fluid out of the tissue, causing significant local geometry change and thus stress concentration. Surface gluing methods can only hold a thin layer of tissue due to the very low shear stiffness of the tissues.

To hold such soft tensile specimens in the test sections, cryogenic methods have been used to freeze the fluid in the tissue inside the gripping area such that mechanical gripping forces can be applied [72]. The ends of the tensile tissue specimen can also be dried up to have sufficient stiffness for gripping actions without introducing severe stress concentrations [73]. Spikes have been introduced to gripping surfaces to hold the entire specimen cross-sections to move together, thus avoiding non-uniform deformation in the gage section $[67,74]$. Due to the irregular shapes of soft tissue specimens and the difficulties to grip the specimens without causing excessive damage or severe stress concentrations, innovative gripping methods are still in demand. Figure 5 illustrates a gripping method where two pairs of circular bend grips with spikes holding a tubular 


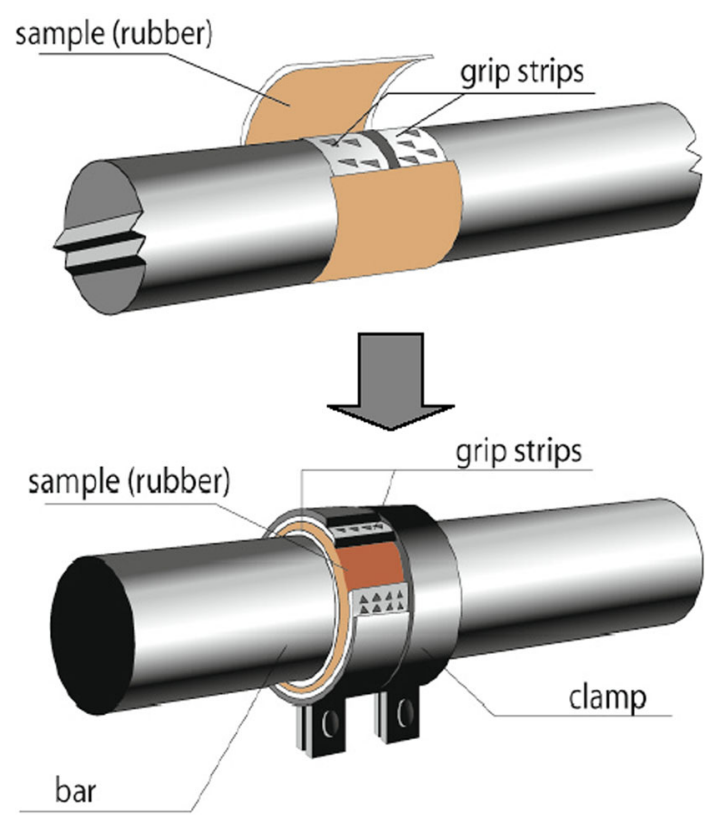

Fig. 5 A gripping method for soft specimens preventing slippage and minimizing inertial effects

soft specimen in the test section. The tubular configuration also minimizes radial inertia effects, as discussed in the next section.

\section{Challenge: Inertial Effects}

In a Kolsky-bar experiment, the specimen is initially at rest. Stress wave accelerates the strain rate from zero to a desired level. During this acceleration process, inertia-induced stresses are encountered in both axial and transverse directions. In addition, even after the axial strain rate reaches a constant level, non-linear axial-transverse geometric relations can induce radial inertia stresses [75]. These inertia-induced stresses in the specimen are coupled to the stresses in the axial direction through Poisson's effect or incompressibility and are measured as part of the transmitted signal [Eq. (7)], which is mixed up with the mechanical response of the specimen material. This is not the purpose of the experiment designed to measure only the mechanical response. In experiments on conventional materials, the inertial effects are typically negligible compared to the mechanical response [75]. However, when the specimen is very soft, inertial effects can dominate over the mechanical response, leading to erroneous results. Therefore, inertial effects are part of the experimental design to obtain valid results. This inertia-induced complexity is the characteristic that distinguishes dynamic material characterization problems from quasi-static tests and should be addressed property in dynamic experimental designs to obtain accurate results.

\section{Remedy: Inertia Minimization Methods}

For a Kolsky bar experiment conducted under commonly used testing conditions (a specimen of a diameter of about $10 \mathrm{~mm}$, with a density similar to water, deforming at a strain rate in the order of $10^{3} \mathrm{~s}^{-1}$ ), the inertia-induced stresses are in the order of 1-2 MPa [76], which would be considered negligible when characterizing common engineered materials even as soft as a PMMA (Plexiglas, yielding around $100 \mathrm{MPa}$ ). However, for soft materials whose strength is in the order of $1 \mathrm{MPa}$ or below (commonly seen in soft tissues), the inertia-induced "noise" in the measured specimen stress histories overshadows the mechanical responses of the soft specimens [77, 78]. Innovative experimental solutions must be developed to delineate the stress-strain response of the specimen material.

To overcome the axial acceleration, specimen thickness for soft materials has always been thin since Kolsky [6]. When the specimen is under axial stress equilibrium, inertia-induced stress in the axial direction becomes too small to be noticed. Inertial stress in the radial direction is zero on the specimen edge and gradually accumulates to a higher level towards the center [75]. Because of this distribution of the transverse inertial stress, a specimen can be made hollow to open up another stress-free surface. The combined use of such washer-shaped specimen geometry (to avoid high-amplitude inertial stress accumulation) and pulse shaping (to avoid initial peak in deformation acceleration) has been proven to be effective to minimize inertial stress in the characterization of soft tissues such as muscles [78]. Figure 6 illustrates the radial inertia-induced extra stress distribution in a solid circular specimen on the top left. The photograph of a gel rubber specimen shows the washer-shaped configuration on the top right. The lower figure shows a comparison of the inertia-induced radial stress distributions in a solid and a hollow specimen.

If the specimen material is even softer such as brain tissue and lung tissue, the inertial stress will dominate over the stress-strain responses since the deformation-induced stresses are two orders of magnitude smaller than the inertial stresses [77, 78]. In this case, the shear response of the material is orders of magnitude smaller than the bulk modulus, the attempt to characterize the materials under uniaxial stress conditions at high rates of deformation becomes infeasible. The bulk and shear portions of the mechanical responses may be characterized separately [79]. However, the low shear wave speeds in such extra soft materials limit the opportunity of dynamic stress equilibrium which depends on stress wave propagation and reverberation. Hybrid experiments combining physical experiments such as dynamic torsion experiments and numerical modeling may be an effective approach to determine the dynamic shear properties of such materials. 

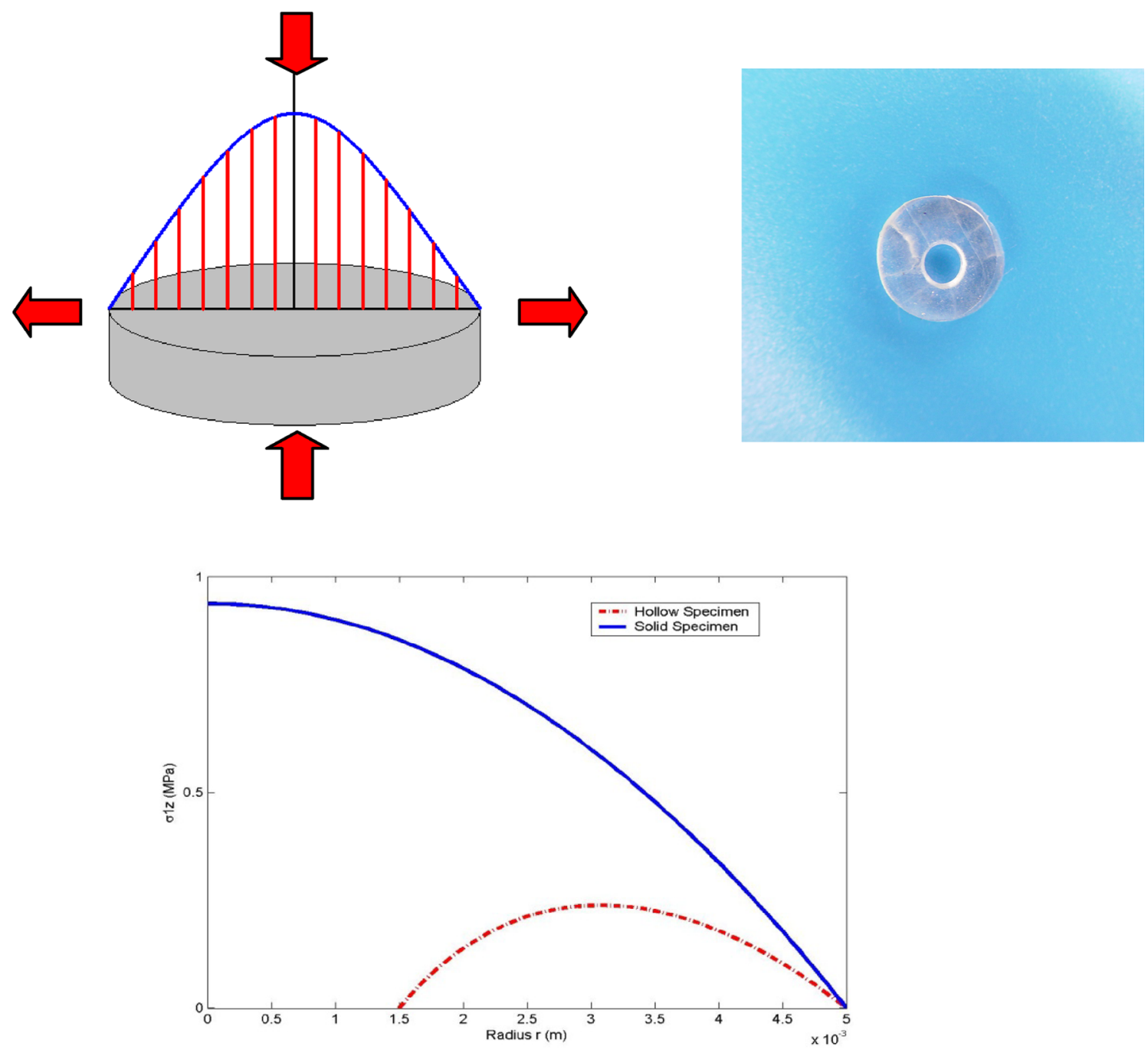

Fig. 6 Inertia induced radial stress distribution in a solid disk specimen (upper left); a washer-shaped specimen (upper right); and the inertia induced stress distributions in a solid and a hollow specimen (lower)

\section{Challenge: Deformation and Damage Visualization}

In many applications of soft materials, post-damage and post-failure behaviors are important energy-dissipation processes and are important to identify and understand. Thus, monitoring dynamic damage initiation and propagation becomes an important aspect in the design of experiments for dynamic material property characterization. High-speed surface measurements have also been developed [22-25]. However, the damage history inside opaque material cannot be recorded.

\section{Remedy: Real-Time Visualization Techniques}

One way to see the damage evolution is to stop the dynamic load at various stages and section of the specimen to identify internal damage. For this purpose, single-loading techniques have been developed to recover the specimen after a known loading history for corresponding microstructure characterization [51, 52]. In such experiments, the dynamic loading on the specimen is stopped at a pre-determined load level. Any repeated stress-wave loading on the specimen that are commonly encountered in conventional Kolsky bar experiments is prevented [52, 64].

Since the dynamic damage process is dynamic in nature, the stop of dynamic loading does not necessarily stop the damage development immediately. Therefore, the damage state revealed on the recovered specimen may not correspond to the loading history precisely. To understand the dynamic damage process more accurately, real-time damage visualization methods are desired. Recently, Kolsky bars have been integrated with high-speed, high-resolution $\mathrm{X}$-ray phase contract imaging (PCI) and X-ray diffraction (XRD) methods to realize this desired capability [16-21]. The high-speed X-ray PCI and XRD can also be used simultaneously to reveal the dynamic deformation and 
Fig. 7 A schematic of the Kolsky bar experimental setup with simultaneous high-speed $\mathrm{X}$-ray phase contrast imaging and X-ray diffraction

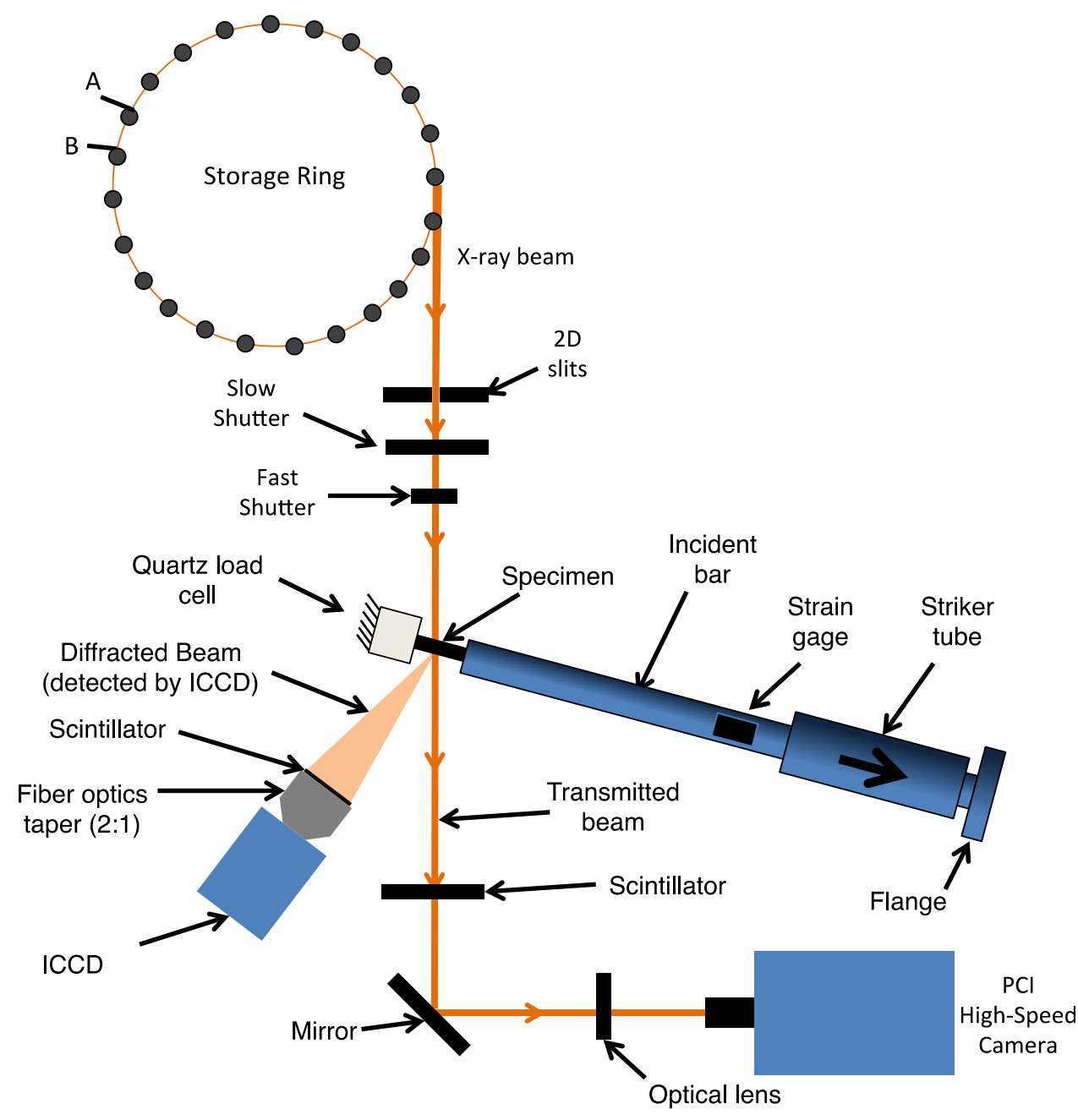

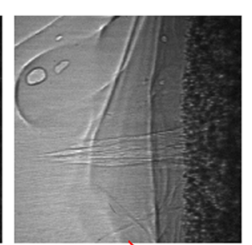
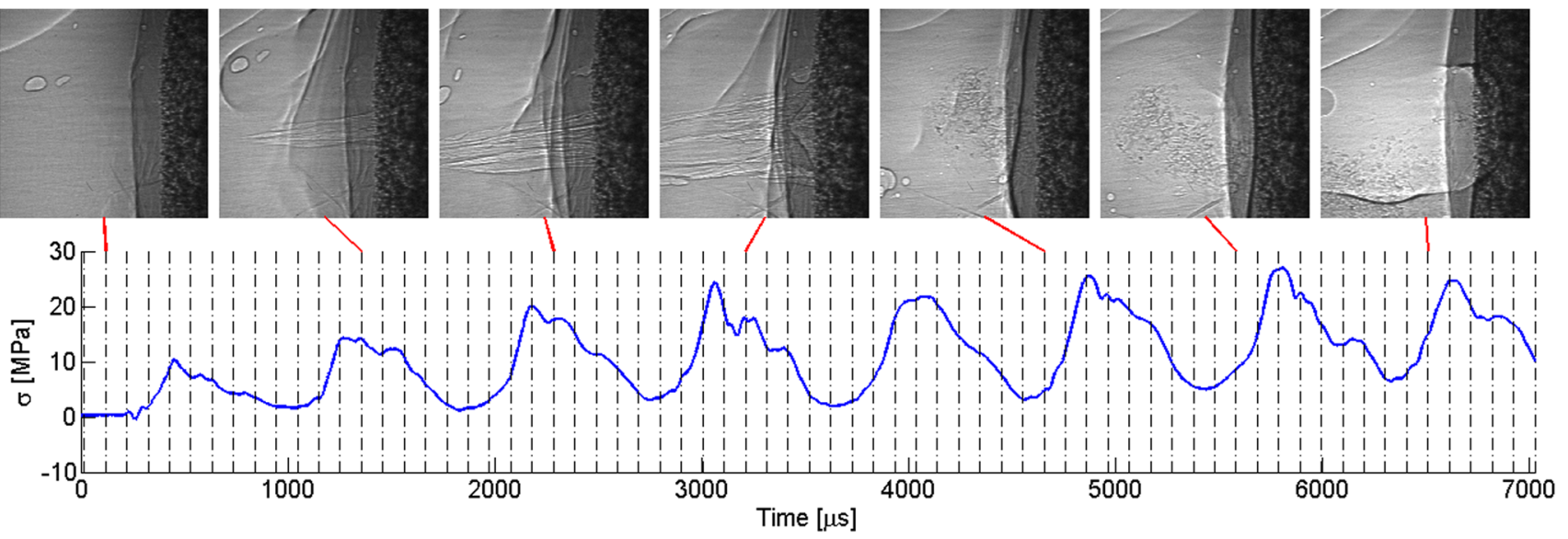

Fig. 8 High-speed phase contact images recording the dynamic damaging process at a ligament-bone joint with corresponding loading history

damage processes from different angles at different length scales in a single dynamic experiment [19]. Figure 7 shows a schematic of the experimental setup where a Kolsky bar is placed at a slight angle from the perpendicular direction of the synchrotron X-ray beam [19]. The high-speed imaging system directly down the beamline is the phase contrast imaging recording the dynamic deformation through the specimen thickness. The camera at an angle 
records the high-speed XRD simultaneously. Figure 8 shows a series of high-speed PCI images of a ligament getting pulled off horizontally from a bone joint at dynamic rates [80]. The bone is the dark object on the right side of each image. Even under the intense X-ray, the damage in the soft ligament is clearly seen. The dynamic damage history is also corresponding to the pulling load history.

\section{Summary}

When Kolsky bars are used to characterize the dynamic behavior of soft materials, weak transmitted signal, dynamic stress equilibrium, constant strain-rate deformation, experiments at intermediate strain rates, specimen gripping methods, inertia effects, and dynamic damage visualization post major challenges in obtaining accurate mechanical responses for soft materials. Modifications to Kolsky bars, especially those over the recent decade, are briefly summarized to address each of the challenges for soft-material characterization in this paper.

Acknowledgments The author's time writing this article was partially sponsored by the Army Research Laboratory and was accomplished under Cooperative Agreement Number W911NF-12-2-0022. The views and conclusions contained in this document are those of the authors and should not be interpreted as representing the official policies, either expressed or implied, of the Army Research Laboratory or the U.S. Government. The U.S. Government is authorized to reproduce and distribute reprints for Government purposes notwithstanding any copyright notation herein.

\section{References}

1. Gray GT, Blumenthal WR (2000) Split Hopkinson pressure bar testing of soft materials. Mech Test Eval, ASM Metals Handb, Am Soc Metals 8:488-496

2. Song B, Chen W, Frew DJ (2004) Quasi-static and dynamic compressive and failure behaviors of an epoxy syntactic foam. J Compos Mater 38:915-936

3. Van Sligtenhorst C, Cronin DS, Brodland GW (2006) High strain rate compressive properties of bovine muscle tissue determined using a split Hopkinson bar apparatus. J Biomech 39:1852-2858

4. Chen W, Song B (2009) Dynamic characterization of soft materials. In: Shukla A, Ravichandran G, Rajapakse Y (eds) Chapter 1 in dynamic failure of materials and structures. Springer, New York, pp 1-28

5. Song B, Chen W (2005) Split Hopkinson bar techniques for characterizing soft materials. Lat Am J Solids Struct 2:113-152

6. Kolsky H (1949) An investigation of the mechanical properties of materials at very high rates of loading. Proc Phys Soc Lond B62:676-700

7. Chen W, Song B (2011) Split Hopkinson (Kolsky) bar: design, testing, and applications. Springer, New York

8. Nie X, Sanborn B, Weerasooriya T, Chen W (2013) High-rate bulk and shear responses of bovine brain tissue. Int J Impact Eng 53:56-61

9. Casem DT, Grunschel SE, Schuster BE (2012) Normal and transverse displacement interferometers applied to small diameter Kolsky bars. Exp Mech 52:173-184
10. Brown EN, Rae PJ, Orler EB (2006) The influence of temperature and strain rate on the constitutive and damage responses of polychlorotrifluoroethylene (PCTFE, Kel-F 81). Polymer 47(21):7506-7518

11. Rae PJ, Brown EN, Orler EB (2007) The mechanical properties of poly(ether-ether-ketone) (PEEK) with emphasis on the large compressive strain response. Polymer 48(2):598-615

12. Jordan JL, Siviour CR, Foley JR, Brown EN (2007) Compressive properties of extruded polytetrafluoroethylene. Polymer 48(14):4184-4195

13. Furmanski J, Trujillo CP, Martinez DT, Gray GT III, Brown EN (2012) Dynamic-tensile-extrusion for investigating large strain and high strain rate behavior of polymers. Polym Test 31(8):1031-1037

14. Siviour CR (2009) A measurement of wave propagation in the split Hopkinson pressure bar. Meas Sci Technol 20(6):065702

15. Nakai K, Yokoyama T (2015) Uniaxial compressive response and constitutive modeling of selected polymers over a wide range of strain rates. J Dyn Behav Mater 1:15-27

16. Hudspeth M, Claus B, Dubelman S, Black J, Mondal A, Parab N, Funnell C, Hai F, Qi ML, Fezzaa K, Luo SN, Chen W (2013) High speed synchrotron X-ray phase contrast imaging of dynamic material response to split Hopkinson bar loading. Rev Sci Instrum 84:025102

17. Parab N, Claus B, Hudspeth M, Black J, Mondal A, Sun J, Fezzaa K, Xiao X, Luo SN, Chen W (2014) Experimental assessment of fracture of individual sand particles at different loading rates. Int J Impact Eng 68:8-14

18. Lu L, Fan D, Bie B, Ran X, Qi M, Parab N, Sun J, Liao H, Hudspeth M, Claus B, Fezzaa K, Sun T, Chen W, Gong X, Luo S (2014) Dynamic strain field mapping with synchrotron X-ray digital image correlation. Rev Sci Instrum 85:076101

19. Hudspeth M, Sun T, Parab N, Guo Z, Fezzaa K, Luo S, Chen W (2015) Investigation of material deformation mechanisms during high-rate loading via simultaneous X-ray diffraction and phase contrast imaging. J Synchron Radiat 22:49-58

20. Hudspeth M, Claus B, Parab N, Lim B, Sun J, Chen W, Fezzaa K (2015) In situ visual observation of fracture processes in several high-performance fibers. J Dyn Behav Mater 1:55-64

21. Li A, Fan D, Lu L, Huang JY, Zhao JCEF, Qi ML, Sun T, Fezzaa K, Xiao XH, Suo T, Chen W, Li YL, Zhu MH, Luo SN (2015) Dynamic fracture of $\mathrm{C} / \mathrm{SiC}$ composites under high strain-rate loading: microstructures and mechanisms. Carbon 91:468-478

22. Pierron F, Grediac M (2012) Virtual fields method: extracting constitutive mechanical parameters from full field deformation measurements. Springer, New York

23. Pierron F, Forquin P (2012) Ultra-high-speed full-field deformation measurements on concrete spalling specimens and stiffness identification with the virtual fields method. Strain 48(5):388-405

24. Chu TC, Ranson WF, Sutton MA (1985) Applications of digitalimage-correlation techniques to experimental mechanics. Exp Mech 25(3):232-244

25. Gilat A, Schmidt TE, Walker AL (2009) Full field strain measurement in compression and tensile split Hopkinson bar experiments. Exp Mech 49(2):291-302

26. Zhao H, Gary G (1997) A new method for the separation of waves: application to the SHPB technique for an unlimited duration of measurement. J Mech Phys Solids 45:1185-1202

27. Park SW, Zhou M (1999) Separation of elastic waves in split Hopkinson bars using one-point strain measurements. Exp Mech 39:287-294

28. Chen W, Zhang B, Forrestal MJ (1999) A split Hopkinson bar technique for low-impedance materials. Exp Mech 39:81-85

29. Chen W, Lu F, Frew DJ, Forrestal MJ (2002) Dynamic compression testing of soft materials. ASME Trans J Appl Mech 69:214-223 
30. Bateman VI, Brown FA (1997) The use of a beryllium Hopkinson bar to characterize in-axis and cross-axis accelerometer response in shock environments. Sandia National Laboratories report SAND-97-2862C

31. Bateman VI, Leisher WB, Brown FA, Davie NT (1993) Calibration of a Hopkinson bar with a transfer standard. Shock Vib 1(2): $145-152$

32. Siviour CR, Gifford MJ, Walley SM, Proud WG, Field JE (2004) Particle size effects on the mechanical properties of a polymer bonded explosive. J Mater Sci 39(4):1255-1258

33. Wang L, Labibes K, Azari Z, Pluvinage G (1994) Generalization of split Hopkinson bar technique to use viscoelastic bars. Int J Impact Eng 15:669-686

34. Zhao H, Gary G, Klepaczko JR (1997) On the use of a viscoelastic split Hopkinson pressure bar. Int $\mathrm{J}$ Impact Eng 19:319-330

35. Bacon C (1998) An experimental method for considering dispersion and attenuation in a viscoelastic Hopkinson bar. Exp Mech 38:242-249

36. Rao S, Shim VPW, Quah SE (1997) Dynamic mechanical properties of polyurethane elastomers using a nonmetallic Hopkinson bar. J Appl Polym Sci 66:619-631

37. Casem DT, Fourney WL, Chang P (2003) A polymeric split Hopkinson pressure bar instrumented with velocity gages. Exp Mech 43:420-427

38. Kown J, Subhash G (2010) Compressive strain rate sensitivity of ballistic gelatin. J Biomech 43:420-425

39. Zhao H, Gary G (1995) A three dimensional analytical solution of the longitudinal wave propagation in an infinite linear viscoelastic cylindrical bar: application to experimental techniques. J Mech Phys Solids 43:1335-1348

40. Werner BT, Daniel IM (2014) Characterization and modeling of polymeric matrix under multi-axial static and dynamic loading. Compos Sci Technol 102:113-119

41. Shergold OA, Fleck NA, Radford D (2006) The uniaxial stress versus strain response of pig skin and silicone rubber at low and high strain rates. Int J Impact Eng 32:1384-1402

42. Chen W, Lu F, Zhou B (2000) A quartz-crystal-embedded split Hopkinson pressure bar for soft materials. Exp Mech 40:1-6

43. Hudspeth M, Nie X, Chen W, Lewis R (2012) Effect of loading rate on mechanical properties and fracture morphology of spider silk. Biomacromolecules 13:2240-2246

44. Hudspeth M, Nie X, Chen W (2012) Dynamic failure of Dyneema SK76 single fibers under biaxial shear/tension. Polymer 53:5568-5574

45. Rice DA (1983) Sound speed in pulmonary parenchyma. J Appl Physiol 54:304-308

46. Yen RT, Fung YC, Ho HH, Butterman G (1986) Speeds of stress wave propagation in lung. J Appl Physiol 61:701-705

47. Gray GT (2000) Classic split-Hopkinson pressure bar testing. Mech Test Eval Metals Handb, Am Soc Metals 8:462-476

48. Casem DT, Weerasooriya T, Moy P (2005) Inertial effects of quartz force transducers embedded in a split Hopkinson pressure bar. Exp Mech 45:368-376

49. Song B, Chen W (2004) Dynamic stress equilibration in split Hopkinson pressure bar tests on soft materials. Exp Mech 44:300-312

50. Dioh NN, Leevers PS, Williams JG (1993) Thickness effects in split Hopkinson pressure bar tests. Polymer 34:4230-4234

51. Song B, Chen WW, Dou S, Winfree NA, Kang JH (2005) Strainrate effects on elastic and early cell-collapse responses of a polystyrene foam. Int J Impact Eng 31:509-521

52. Nemat-Nasser S, Isaacs JB, Starrett JE (1991) Hopkinson techniques for dynamic recovery experiments. Proc R Soc Lond 435:371-391

53. Frew DJ, Forrestal MJ, Chen W (2002) Pulse shaping techniques for testing brittle materials with a split Hopkinson pressure bar. Exp Mech 42:93-106
54. Frew DJ, Forrestal MJ, Chen W (2005) Pulse shaping techniques for testing high-strength steel with a split Hopkinson pressure bar. Exp Mech 45:186-195

55. Zhao H, Gary G (2002) Behaviour characterization of polymeric foams over a large range of strain rates. Int J Veh Des 30:135-145

56. DT Casem (2010) Hopkinson bar pulse-shaping with variable impedance projectiles - an inverse approach for projectile design. Army Research Laboratory technical report ARL-TR-5246

57. Song B, Syn CJ, Grupido CL, Chen W, Lu W-Y (2008) A long split Hopkinson pressure bar (LSHPB) for intermediate-rate characterization of soft materials. Exp Mech 48:807-815

58. Deschanel S, Greviskes BP, Bertoldi K, Sarva SS, Chen W, Samuels SL, Cohen RE, Boyce MC (2009) Rate dependent finite deformation stress-strain behavior in ethylene methacrylic acid copolymer and an ethylene methacrylic acid butyl acrylate copolymer. Polymer 50:227-235

59. Sarva S, Deschanel S, Boyce MC, Chen W (2007) Stress-strain behavior of a polyurea and a polyurethane from low to high strain rates. Polym Commun 48:2208-2213

60. Shim J, Mohr D (2009) Using split Hopkinson pressure bar to perform large strain compression tests on polyurea at low, intermediate and high strain rates. Int $\mathbf{J}$ Impact Eng 36:1116-1127

61. https://www.mts.com/en/products/producttype/test-systems/loadframes-uniaxial/servohydraulic/high-rate/index.htm

62. http://www.instron.com/en-us/products/testing-systems/dynamicand-fatigue-systems/8800-high-strain-rate

63. Boyce BL, Dilmore MF (2009) The dynamic tensile behavior of tough, ultrahigh-strength steels at strain-rates from $0.0002 \mathrm{~s}^{-1}$ to $200 \mathrm{~s}^{-1}$. Int J Impact Eng 36:263-271

64. Song B, Chen W (2004) Loading and unloading SHPB pulse shaping techniques for dynamic hysteretic loops. Exp Mech 44:622-627

65. Chen W, Lu F, Cheng M (2002) Tension and compression tests of two polymers under quasi-static and dynamic loading. Polym Test 21:113-121

66. Nie X, Song B, Ge Y, Chen WW, Weerasooriya T (2009) Dynamic tensile testing of soft materials. Exp Mech 49:451-458

67. Nie X, Cheng J-I, Chen W, Weerasooriya T (2011) Dynamic tensile response of porcine muscle. J Appl Mech 78:021009

68. Pervin F, Chen W (2009) Dynamic mechanical response of bovine gray matter and white matter brain tissues under compression. J Biomech 42:731-735

69. Pervin F, Chen W, Weerasooriya T (2011) Dynamic compressive response of bovine liver tissue. J Mech Behav Biomed Mater 4:76-84

70. Song B, Ge Y, Chen W, Weerasooriya T (2007) Dynamic and quasi-static compressive response of a porcine muscle. J Biomech 40:2999-3005

71. Pervin F, Chen W, Weerasooriya T (2010) Dynamic compressive response of renal cortex. Int J Struct Chang Solids 2:1-7

72. Cheng M, Chen W (2003) Experimental investigation of the stress-stretch behavior of EPDM rubber with loading rate effects. Int J Solids Struct 40:4749-4768

73. Cheng M, Chen W, Weerasooriya T (2009) Mechanical behavior of bovine tendon with stress-softening and loading rate effects. Adv Theor Appl Mech 2:59-74

74. Lim J, Hong J, Chen W, Weerasooriya T (2011) Mechanical response of pig skin under dynamic tensile loading. Int J Impact Eng 38:130-135

75. Forrestal MJ, Wright TW, Chen W (2007) The effect of radial inertia on brittle samples during the split Hopkinson pressure bar test. Int J Impact Eng 34:405-411

76. Warren TL, Forrestal MJ (2010) Comments on the effects of radial inertia in the Kolsky bar test for an incompressible material. Exp Mech 50:1253-1255 
77. Sanborn B, Nie X, Chen W, Weerasooriya T (2013) High strainrate pure shear and uniaxial compressive response of porcine lung tissue. J Appl Mech 80:011029

78. Song B, Ge Y, Chen W, Weerasooriya T (2007) Radial inertia effects in Kolsky bar testing of extra-soft specimens. Exp Mech 47:659-670
79. Saraf H, Ramesh KT, Lennon AM, Merkle AC, Roberts JC (2007) Mechanical properties of soft human tissues under dynamic loading. J Biomech 40:1960-1967

80. Claus B (2014) Characteristics of fibrous tissue at high rates of tensile loading. MS thesis, Purdue University, West Lafayette, Indiana 